\title{
Comparing perceptual and preferential decision making
}

\author{
Gilles Dutilh $^{1} \cdot$ Jörg Rieskamp ${ }^{1}$
}

Published online: 2 October 2015

(C) Psychonomic Society, Inc. 2015

\begin{abstract}
Perceptual and preferential decision making have been studied largely in isolation. Perceptual decisions are considered to be at a non-deliberative cognitive level and have an outside criterion that defines the quality of decisions. Preferential decisions are considered to be at a higher cognitive level and the quality of decisions depend on the decision maker's subjective goals. Besides these crucial differences, both types of decisions also have in common that uncertain information about the choice situation has to be processed before a decision can be made. The present work aims to acknowledge the commonalities of both types of decision making to lay bare the crucial differences. For this aim we examine perceptual and preferential decisions with a novel choice paradigm that uses the identical stimulus material for both types of decisions. This paradigm allows us to model the decisions and response times of both types of decisions with the same sequential sampling model, the drift diffusion model. The results illustrate that the different incentive structure in both types of tasks changes people's behavior so that they process information more efficiently and respond more cautiously in the perceptual as compared to the preferential task. These findings set out a perspective for further integration of perceptual and preferential decision making in a single ramework.
\end{abstract}

Gilles Dutilh

gilles.dutilh@gmail.com

1 Department of Psychology, University of Basel, Missionsstrasse 62a, 4055 Basel, Switzerland
Keywords Sequential sampling models · Risky choice . Value based decisions

\section{Introduction}

People make many decisions every day. Perceptual decisions, such as judging whether a traffic light is green or red are at a low cognitive level as they are made rather quickly without much deliberation. Preferential decisions, such as buying a car or investing in stocks, are at a higher cognitive level as they require more deliberation and depend on the decision maker's goals. Despite these differences, the two types of decisions also have a lot in common: in both situations, information about the choice options needs to be considered and processed to form a final decision. The present work aims at identifying the crucial differences between the two types of decision making.

\section{Perceptual decision making}

The first type of decision making, perceptual decision making, has been the focus of psychological research for decades. This research aims at understanding how people process objective information to make a decision. For this goal, many experimental tasks have been developed. For example, in the popular moving dot motion task, participants have to make the elementary perceptual decision as to whether dots are moving to the left or right (e.g., 
Britten, Shadlen, Newsome \& Movshon, 1992). More complicated tasks include lexical decision, in which participants have to decide quickly whether a presented letter string is an existing word or not (e.g., Keuleers, Diependaele \& Brysbaert, 2010) and the house-face discrimination task in which participants have to judge whether presented pictures represent either houses or faces (e.g., Summerfield, Egner, Mangels \& Hirsch, 2006). Arguably, the latter two tasks require more than just perceptual information processing. We use the term "perceptual" here to indicate tasks where there is an objective criterion that defines the correctness of the response. The performance of participants on perceptual tasks, as expressed in response time and accuracy, is assumed to reflect the ease of processing of stimulus information: fast and accurate responding indicates efficient information processing; slow and inaccurate responding indicates less efficient processing. This straightforward interpretation of speed and accuracy is, however, often problematic due to the strong relation between accuracy and response time (RT). This relation is known as the speedaccuracy trade-off (Schouten \& Bekker, 1967; Pachella, 1974; Wickelgren, 1977): speeding up leads to more errors and more accurate behavior requires slower responding. A very successful account of RT data is offered by the diffusion model (Ratcliff, 1978; Ratcliff \& Tuerlinckx, 2002), which naturally models the speed-accuracy trade-off by the adjustment of response thresholds.

\section{The diffusion model for perceptual decision making}

The diffusion model has been applied mostly to speeded two-choice situations where there is one correct response option (for an exception, see Milosavljevic, Malmaud, Huth, Koch \& Rangel, 2010). The range of tasks that have been modeled with the diffusion model include lexical decision (Wagenmakers, Ratcliff, Gomez \& McKoon, 2008; Dutilh, Krypotos \& Wagenmakers, 2011), letter discrimination (Thapar, Ratcliff \& McKoon, 2003), brightness discrimination (Ratcliff and Rouder, 1998, 2000), the random dot motion task (van Ravenzwaaij, Dutilh \& Wagenmakers, 2012), and the implicit association test (Van Ravenzwaaij, Van der Maas \& Wagenmakers, 2011).

The diffusion model assumes that when presented with a stimulus, participants continuously sample information from that stimulus. When the stimulus is of, say, type "A", the integrated evidence in favor of response A over $B$ will on average increase over time, until a pre-set boundary is reached. At this point, the corresponding response is initiated (Fig. 1). However, the integrated evidence is assumed to be noisy. This assumption allows the model to account for both variability in response times and the occurrence of eventual errors (dark line in Fig. 1).
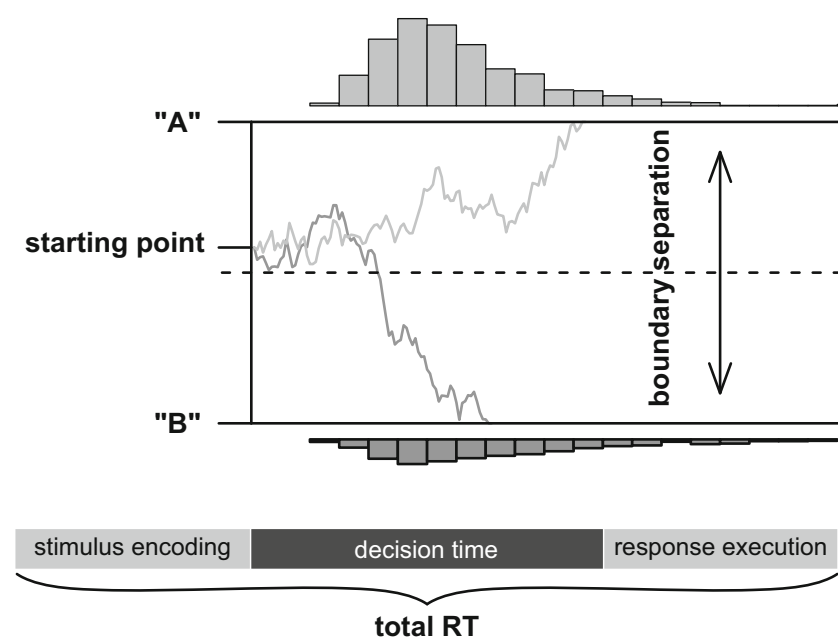

Fig. 1 The diffusion model. The two example sample paths represent the accumulation of evidence from an a stimulus, resulting in one correct response (light line) and one error response (dark line). A response is initiated when one of the boundaries is reached. The distributions of correct and error responses are represented by the histograms below and above the boundary respectively. As is evident from the histograms, the correct, (upper) a boundary is reached more often than the incorrect, (lower) b boundary. The total RT consists of the sum of a decision component, modeled by the diffusion process, and a non-decision component that represents the time needed for peripheral processes such as stimulus encoding and response execution

The diffusion process is governed by seven parameters. The parameters of the model are 1) drift rate $v$, which quantifies the rate of information processing. Low absolute values of $v$ produce relatively long RTs and high error rates and are associated with difficult stimuli or low ability. 2) Boundary separation a defines how much evidence is integrated before a response is initiated. Higher boundary values yield relatively slow, but accurate responding. Lower boundary values yield relatively fast, but error-prone responding. The magnitude of the boundary separation thus reflects response caution and thereby defines the speedaccuracy trade-off. 3) Starting point $z$ reflects eventual a priory bias in favor of one of the response options. Often, studies refer to relative bias $B$, which is defined as $z / a$. A value of .5 for bias $B$ reflects unbiased responding. 4) Non-decision time $T_{e r}$ defines the shift of the response time distributions. This parameter is assumed to capture stimulus-nonspecific components of RT, such as response preparation and motor execution. In addition, there are three parameters that define the across-trial variability of three of the parameters. 5) $\eta$ : The drift rate on each trials is assumed to be drawn from a normal distribution with mean $v$ and standard deviation $\eta$. 6) $s_{z}$ : Each trial's starting point is assumed to be drawn from a uniform distribution around mean $z$ with range $s_{z}$. Parameters $\eta$ and $s_{z}$ allow the 
diffusion model to account for the fact that errors are sometimes faster and sometimes quicker than correct responses. 7) $s_{t}$ : The non-decision time on each trial is assumed to be drawn from a uniform distribution around mean $T_{e r}$ with range $s_{t}$. This variability in non-decision time allows the model to account for relatively large variability in the leading edge (e.g., the $10 \%$ quantile) of the response time distribution.

The big strength of the diffusion model is that its parameters, when estimated to fit a data set, can be interpreted as reflecting psychological components of the decision process from which these data originate. Validation studies have shown that specific experimental manipulations affect the parameters according to the interpretation given in the descriptions above. Studies by Voss, Rothermund \& Voss (2004), Ratcliff \& Rouder (1998) and Wagenmakers et al. (2008) have shown that instructions to respond less cautiously lead to lower estimates of boundary separation $a$, easier stimuli lead to higher estimates of drift rate $v$, unequal reward rates and unequal proportions of stimulus types influence the estimate of bias $B$, and a less convenient response-button mapping lead to an increased non-decision time $T_{e r}$. Note that this unique mapping of parameters to decision components have been challenged. For example, Rae et al. (2014) show that increased pressure on speed might not only reduce boundary separation, but also the quality of information processing, as quantified by drift rate $v$.

The diffusion model is a typical sequential sampling model. The simple assumption of a sequential sampling process allows the model to describe many phenomena in empirical data. This assumption has also proven able to account for data in the second type of decision making that we study here: preferential decision making.

\section{Preferential decision making}

Preferential decision making is an important topic of study in both psychology and economics. Studies on preferential decision making aim at understanding how people trade off the different attributes of choice options to come to a decision. For example, in research on consumer behavior, participants are asked to choose between different products in order to identify the importance they give to the different attributes (e.g., Pettibone \& Wedell, 2000). To study preferential decision making under risk, participants are often confronted with choices between monetary gambles (e.g., Holt \& Laury, 2002). Monetary gambles are well specified by a set of potential outcomes and the respective probabilities with which these occur. When choosing between gambles, people trade-off the expected value of the gambles' potential outcomes and the gambles' risk, which is often characterized by the variance of the outcomes. The way people trade-off risk and expected value reflects their risk attitude; When choosing between two gambles with equal expected value, a risk-averse decision maker chooses the option with the lower variance, whereas a risk loving decision maker prefers the option with the higher variance.

In some risky choice situations, the probabilities with which outcomes occur are not specified. These situations are often labeled as decisions under uncertainty (Busemeyer, 1985). In such situations, decision makers' preferences also depend on their attitude towards ambiguity (Ellsberg, 1961). People are considered ambiguity-averse when they prefer a gamble about which the outcome distribution is known over a gamble with an unknown outcome distribution.

To predict people's decisions under risk, various models have been suggested. The standard economic modeling approach assumes that people's choices are consistent with normative choice principles. If people obey these principles, it is possible to construct a utility function, so that people always choose the option with the maximum expected utility (von Neumann \& Morgenstern, 1945; Savage, 1954). Such expected utility theories were shown to have problems predicting some key phenomena in risky decision making. To offer a more flexible account for preferences under uncertainty, revised utility models were proposed, most importantly prospect theory (Kahneman \& Tversky, 1979; Tversky \& Kahneman, 1992).

Besides these descriptive approaches, researchers have worked on approaches that describe the cognitive process that leads to a decision (e.g., Weber \& Johnson, 2009). Among those process models, sequential sampling models play a prominent role (for an overview, see Rieskamp et al., 2006).

\section{Sequential sampling model for preferential decision making}

Following the work of Ratcliff (1978) and Busemeyer \& Townsend (1993) developed a sequential sampling model for preferential decisions under risk called decision field theory (DFT). DFT assumes that a decision maker integrates relative preference for choice alternatives until a decision threshold is reached. In contrast to the diffusion model, which is silent about the origin of the information that is accumulated over time, DFT specifies how the properties of choice options define the evidence that enters the preference integration process. The process is assumed to be fed by sequential samples from the different outcomes of the different choice options. The sampled outcomes are integrated in a momentary preference state. During the decision process, the attention of the decision maker alternates stochastically between the different outcomes of all choice options. 
Therefore, the preference information fed to the diffusion process differs over time; At one time point during a decision the decision maker focuses on potential losses of the choice options whereas at another time point the potential gains are considered (Diederich \& Busemeyer, 2003). The probability that each outcome is attended to is proportional to the probability with which the outcome occurs. A similar sequential sampling model was proposed by Usher \& McClelland (2004), who suggested an accumulator model of decision making: the leaky, competing accumulator (LCA) model. The most important theoretical difference with DFT is that LCA allows disadvantages of an alternative to loom larger than advantages. Despite their differences, both models predict that people's choices are in nature probabilistic. Furthermore, both models explain various choice phenomena such as preference reversals and dependence on irrelevant choice alternatives (e.g., Rieskamp et al., 2006). Recent research in neuroscience indicates that the assumption of sequential integration of value information is neurologically plausible (e.g., Gold \& Shadlen, 2007; Gluth et al., 2012).

\section{Differences between perceptual and preferential decision making}

The discussion above shows that both in the perceptual and preferential decision making literature, sequential sampling models have been proven successful in accounting for important empirical phenomena. The shared basic assumption of sequential information integration supports the intuition that perceptual and preferential decision making behavior have a lot in common. Beside these similarities however, there are also important differences between both domains.

\section{Criterion for the quality of a response}

The foremost difference between perceptual and preferential decisions lies in how the quality of a decision is defined. In perceptual decision situations, the accuracy of a choice can be determined by an outside criterion. For example, in a random dot motion task, the direction of the movement of the dots is objectively left or right. Although there might be some noise in the information perceived from the stimulus, there is only one correct response to each stimulus. In contrast, in a preferential choice situation, no external criterion exists that defines which response is correct. For example, in a gamble choice task, some participants prefer to play safe and go for a sure gain of $\$ 10$, whereas others like to gamble with a $10 \%$ probability to win $\$ 100$. Thus, the criterion is defined by the decision maker's subjective goals and only in the light of these goals can the accuracy of decisions be evaluated.

\section{Outcome of decisions}

A second property that often differs between perceptual and preferential decision making is the level of certainty of the outcomes. In most perceptual choice situations that have been studied in the literature, the outcome of a decision is determined by the choice: a correct response yields a reward and an incorrect response yields a penalty (for an exception, see Ratcliff \& Rouder, 1998). In contrast, in preferential choice situations under risk, the outcome of a decision depends on chance: the outcome of playing a gamble that leads to a gain of $\$ 100$ with a probability of $\$ 90 \%$ (zero otherwise) might turn out better or worse than a sure $\$ 10$. Thus, in these risky preferential choice situations, the choice alternatives differ by the certainty with which the outcomes occur, i.e., the amount of risk involved.

\section{Aims of this study}

Despite these differences, the success of the same type of models to describe people's behavior in two different domains, suggests an elemental similarity between perceptual and preferential decisions. In line with this observation, Gold \& Shadlen (2007) suggest that the same underlying mechanisms govern decisions in the two different domains of decision making. Following this suggestion we aim to describe both types of decision making with one single model, namely the diffusion model. By doing so, we will be able to identify the crucial differences between perceptual and preferential decision making. To be able to apply the diffusion model to both types of decision making, we develop a paradigm that allows us to elicit both perceptual and preferential decision making by using identical task material.

In the following, we will first introduce this "marbles task" and explore various hypotheses about the behavior on the task. Subsequently, we present a study in which participants perform on the perceptual and preferential version of this task. We show the results in terms of RT and accuracy, but also apply the diffusion model to participants' data in both instances of the task. This analysis allows us to lay bare the components of the decision process that underlie the differences between perceptual and preferential decision making.

\section{A unifying paradigm for perceptual and preferential decision making}

The choice paradigm we develop here aims at eliciting both types of decisions by manipulating only those features of a task that are specific for either perceptual or preferential 
decision making. These features are 1) the objective vs. subjective outside criterion and 2) the deterministic vs. probabilistic nature of the outcome. Besides these crucial differences the paradigm should be identical with respect to all other choice and context characteristics.

\section{Principles of the paradigm}

Our paradigm uses the same stimulus to present either a perceptual or a preferential choice: a matrix of 100 black and white dots (Fig. 2a-c). When presented in the perceptual version of the task, participants have to decide whether the matrix contains more black or more white dots. This task is very similar to, e.g., the brightness discrimination task used by Ratcliff \& Rouder (1998). When presented in the preferential version of the task, participants are told that the matrix represents an urn with a total of 100 black and white marbles - each of which has an associated monetary payoff. If the participant chooses to play this urn, a marble will be drawn and the associated payoff is paid out to the participant. Urn paradigms like this have a long tradition in research on decision making under risk (e.g., Phillips \& Edwards, 1966). In our marbles task, participants are asked whether they want to draw from the urn that is represented by the stimulus matrix (target gamble), or prefer playing the reference gamble, which is an urn consisting of 50 black and 50 white marbles (Fig. 2d). When the reference gamble has the same positive and negative payoffs as the presented target gamble, the only difference between the target and the standard gamble is the probability to win and the ambiguity about this probability. However, the payoffs associated with the marbles in the target gamble can be increased, which increases the involved risk. In sum, this marbles task allows us to elicit both perceptual and preferential choice behavior and to manipulate the ambiguity and risk of the presented choice options.

\section{Predictions}

The marbles task allows us to collect both perceptual and preferential choice data to which we can fit the diffusion model. Therefore, we can now explore predictions about how the domain of decision making (perceptual vs. preferential) and the risk of the decisions in the preferential version influence the components of decision making that are postulated by the diffusion model.

\section{Perceptual vs. preferential information}

In the perceptual task, a correct response yields a sure gain and an error yields a sure loss. Thus, the expected reward difference in reward between two response options is the difference between the maximal gain and loss. In the preferential case, a choice for the gamble with the higher expected value not always yields a gain; a choice for the gamble with the lower expected value not always yields a loss. Thus, the expected reward difference between the two preferential choice options is smaller than between the perceptual choice options, making for higher monetary incentives in the perceptual task. The question is which decision component will be affected by this difference. The higher monetary incentives might on the one hand increase response caution as reflected in boundary separation. On the other hand, they might increase participants attention and therefore cause higher drift rates in the perceptual task.

\section{Risk level}

Changing the possible wins and losses of the target gamble in the preferential task allows us to manipulate the risk level of this target gamble. Risk averse participants will dislike the "target" option more and more when its risk is increased relative to the "reference" option's risk level. In terms of the diffusion model, participant's response to increasing risk could be reflected in a bias parameter that shifts more and more towards the "reference gamble" boundary when risk is increased. However, it might as well be the case that the risk level biases the decision process at the input side, i.e., influence the drift rate. This idea is in line with Zeigenfuse, Pleskac \& Liu (2014), who found that riskaverse participants show a lower drift rate for the risky choice option than for the safe choice option. From that, they conclude that risk averse participants weigh information in favor of riskier options as less valuable than information in favor of the safer option.

\section{Ambiguity}

In the experiment, we do not manipulate ambiguity. However, in the preferential task, one crucial factor that discriminates the reference gamble from the target gamble is that for the reference gamble, the probabilities of the outcomes are known (no ambiguity) whereas for the target gamble these probabilities are unknown (ambiguous). This means that, if participants are ambiguity averse, they should have a bias towards the reference gamble when the risk of the reference and the target gamble are equal. This bias might be reflected in the starting point or in the drift rate.

\section{Method}

\section{Participants}

43 participants (35 female) with mean age of 22.9 (SD = 5.2) participated in this study. Participation was rewarded 


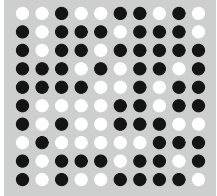

(a)

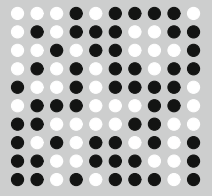

(b)
Fig. 2 a, b, c Stimuli of increasing ratio of white vs. black marbles (40-60, 45-55. 50-50). In the perceptual task, stimuli (a) and (b) require participants to press the button associated with black. In the preferential task, white marbles have a positive value and black marbles have a negative value. Here, a participant who wants to achieve

with either course credit or a monetary show up fee. On top of this base reward, participants got CHF 5 playing money (5 Swiss franc corresponds to roughly \$5). Depending on performance, they could earn up to CHF 5 extra or lose CHF 5. The complete data set is available at the first author's website and at dataverse.org: doi:10.7910/DVN/AOQUAD.

\section{Stimuli}

The stimuli in the preferential and perceptual version of the task were equal. Each stimulus was a square (sides of $86 \mathrm{~mm}$ ) containing $10 \times 10=100$ dots (diameter of 5 $\mathrm{mm}$ ) in black and white on a grey background. Towards the participants, these dots were referred to as marbles. Five different ratios of black and white marbles were presented to the participants (black - white ratios: 40-60, 45-55, 50$50,55-45$, and 60-40). The grid of black and white dots was shuffled for each stimulus (see example stimuli in Fig. 2a-c)

\section{Procedure}

The perceptual and preferential versions of the task were administered within participants and the order of the two conditions was counterbalanced.

\section{Preferential task}

In the preferential task, the five different proportions of white (win) and black (lose) marbles represented the probability of winning the current payoff. Participants chose at each trial whether they wanted to play the target gamble represented by the marble matrix (and press left) or the 50/50 reference gamble (and press right). This reference gamble (Fig. 2d) was shown to them on an instruction screen that preceded each block, but not during the trials. They were instructed that once they had chosen one of the two gambles, one marble were sampled from it. If this marble were white, this yielded a win of the size of the chosen gamble's

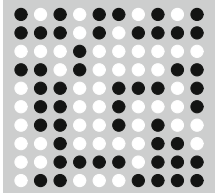

(c)

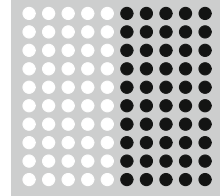

(d) the highest expected value should choose the target gamble over the reference gamble (containing $50 \%$ white and $50 \%$ black marbles) whenever the target gamble contains more than $50 \%$ white marbles (a and b). d The 50-50 reference stimulus shown to participants before each block in the preferential task

payoff. If this marble were black, this yielded a loss of the size of the gamble's payoff.

Trials were presented in 12 blocks of 50 trials, each block containing 10 trials of each black/white ratio. The payoff associated with the white and black marbles of the reference gamble were always plus and minus CHF .50. The payoffs of the target gamble were plus and minus CHF .50 on the first four blocks, 1.50 on the second four blocks, and 2.50 on the last four blocks. The current payoff attached to black and white marbles of the target gamble was shown on the block instruction screen. The payoffs were visualized as one, three or five yellow coins for the white (winning) marbles and one, three or five red coins for the black (loss) marbles. The number of coins thus represented the risk level of the currently presented target gamble relative to the reference gamble. We chose to have a fixed order of risk levels for all participants, so that they first got familiarized with the simplest case, where the payoffs of the target and reference gamble were equal. Below, we refer to the three risk levels as risk level 1,3 , and 5 , referring to 1,3 , and 5 times the basic amount of CHF .50.

There was no direct feedback, but at the end of each 50 trial block, one of the choices was selected at random. This gamble was then played and the resulting reward was presented to the participant.

\section{Perceptual task}

In the perceptual task, participants judged at each trial whether the stimulus contained more black marbles (and press right) or white marbles (and press left). They were instructed to respond as quickly and accurately as possible. The five ratios of black and white marbles yielded three difficulty levels ("easy", "hard" and "impossible", compare Fig. 2a-c). Trials were administered in 4 blocks with each block containing 10 trials of each difficulty level, thus resulting in 200 trials.

Again, no direct feedback was supplied to the participant, but correct responses were silently rewarded a "gain" ticket 

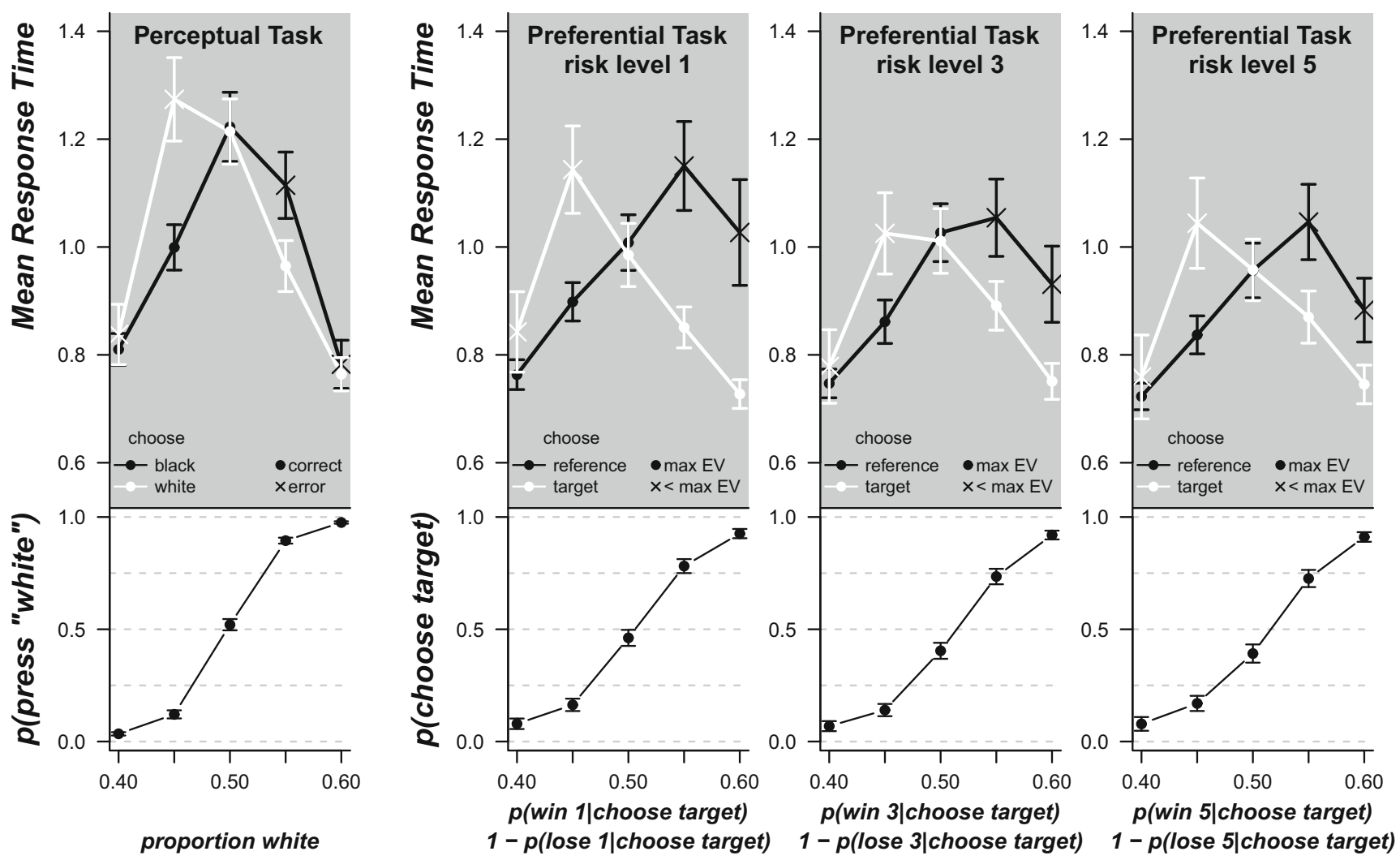

Fig. 3 Mean over participants' median response times and choice proportions for the three main conditions of the experiment (perceptual task and preferential task with three different risk levels). In the

perceptual version of the task, participants respond a bit slower than in the preferential version. The choice proportions are more extreme in the perceptual version of the task

and incorrect responses "loss" ticket. After each block, one of the collected gain and loss tickets was sampled and presented as reward to the participant. The payoff for correct and error responses were CHF .50 and -CHF .50 respectively, matching the potential outcomes in the risk level 1 of the preferential version of the task.

\section{Results}

Below, we first show the descriptive results on RT and choice followed by the results of the diffusion model analyses.

\section{Descriptive results}

Figure 3 shows mean choice probabilities and means over participants' median response times for each cell in the design. Error bars represent standard errors of overparticipant means. The leftmost panels of the figure show data of the perceptual version of the task, the other three panels show the preferential task in which the risk level of the target gamble was 1,3 , and 5 respectively. In the upper figures, white lines present RT for trials where participants pressed the button associated with "more white" or "choose target gamble". Black lines represent mean RT for trials where participants pressed the button associated with "more black" or "choose reference gamble". Solid points indicate majority responses (correct in the perceptual or expected value maximizing in the preferential task). Crosses indicate minority responses (incorrect/expected value minimizing).

\section{Response times}

To analyze which factors influence RT, we performed a comparison of ANOVA models ${ }^{1}$ in which participants' median RTs where regressed on all different combinations of the following factors: (1) condition; perceptual vs. preferential task (2) condition + risk; the factor condition including the three risk levels and (3) stimulus type; the proportion of white marbles in the stimulus, with participants as a random factor. The best performing model was a model including condition and stimulus and their interaction. This

\footnotetext{
${ }^{1}$ The ANOVA models were calculated using the BayesFactor package for R (Morey, Rouder \& Jamil, 2014).
} 
model outperformed models in which the interaction was excluded $(B F=3.10)$ or in which the condition + risk factor was included $\left(B F=1.569 \times 10^{3}\right)$, or in which the main effects condition and stimulus were excluded $(B F=$ $\left.9.430 \times 10^{27}\right)$. This model comparison supports the interpretation that participants are slower in the perceptual than in the preferential task and respond slower to more ambiguous stimuli. The latter effect is more pronounced within the perceptual condition.

\section{Choice proportions}

To analyze which factors influence the choice proportions, we fitted a simple psychometric function (logit) on the choice proportions as a function of the stimuli. We applied a Bayesian Hierarchical method to model the condition effects (the differences between the four panels of the Fig. 3) on the slope (sensitivity) and shift (bias) parameters at the overarching group level. Below, we base our conclusions on the posterior distributions of these overarching parameters.

The figure shows a more pronounced S-shape for the choices in the perceptual than in the preferential task. This finding is supported by $99.9 \%$ of the posterior distribution for the effect slope $e_{\text {perc. }}-$ slope $_{\text {pref.risk } 1}$ lying above zero. This shows that participants respond more deterministically in the perceptual than in the preferential task. Furthermore, the figure suggests that in the preferential task, participants choose the reference stimulus more often for all stimuli. This is supported by $97.4 \%$ of the posterior distribution for the effect shift $t_{\text {perc. }}-$ shift $_{\text {pref.risk } 1}$ lying above zero. This shift of bias increases when the risk level is increased to 3, supported by $99.9 \%$ of the posterior for the effect shif $t_{\text {pref.risk } 3}-$ shif $_{\text {pref.risk } 1}$ lying above zero. No other contrast on slope or shift showed evidence for effects on any of the two parameters.

We want to note here that practice effects might be a confounding factor in our design, in particular since the order of the different risk levels was not counterbalanced. Practice effects could cause more deterministic responding (higher slopes) later in the session and therefore mask eventual effects of risk level. The current design in which the order of the different risk levels was not counterbalanced does not allow to rule out this possibility.

\section{Diffusion model results}

For a better understanding of the descriptive results, we now turn to the diffusion model analysis. We fitted the diffusion model using the DMAT package for MATLAB (Vandekerckhove \& Tuerlinckx, 2007). We applied the chisquare method (Ratcliff \& Tuerlinckx, 2002) using the default fixed bin edges implemented in DMAT. This methods optimizes the match between observed and predicted frequencies in each of the RT bins for correct and error responses.

\section{Model comparison}

To identify the components of the diffusion model that discriminate perceptual from preferential decision making, and to describe how participants' behavior is influenced by different levels of risk, we performed an extensive comparison of diffusion models. In each of these models, we allowed different subsets of parameters to account for the effects of the experimental factors. These models do not represent all possible constellations of parameter restrictions across conditions (this would yield an unfeasible long list of models). Instead, we fit a list of 20 variants that were plausible, in the sense that non-decision time $\left(T_{e r}\right)$ and parameters that are assumed to be pre-set before the decision process $\left(a, z\right.$, and $s_{z}$ ) do not depend on stimulus type. We fitted the full model (model 16 in Table 1) where all plausible effects on parameters were allowed and 19 other models where we constrained parameters across conditions where they did not show consistent effects in the full model. The models that we fitted differ with respect to the parameters that are allowed to vary across the levels of the following factors:

- Condition: Perceptual vs. preferential decisions.

- Risk: The three risk levels for the preferential decisions.

- Stimulus: Proportion of white vs. black marbles in the stimulus matrix.

Note that factor risk level is nested within the second level of factor condition. Table 1 shows which parameters were free to vary with each of the factors listed above. In Table 1 , the contrast $p$ means that the parameter was allowed to vary between the perceptual and preferential task, $r$ means that the parameter was free to vary across the risk levels, $s$ means that the parameter was free to vary across stimulus types (proportion of white/black marbles), and s:sym represents a constrain on drift rates to be symmetrical across stimuli. This constrains drift rates for stimuli that are related to a "left" response to have the same absolute magnitude as drift rates for stimuli related to a "right" response. Under this constraint, there are 3 magnitudes allowed: one for the 40/60 and 60/40 stimuli and one for the $45 / 55$ and $55 / 45$ stimuli and one for the $50 / 50$ stimuli.

To compare the models, we calculated each model's Bayesian Information Criterion (BIC), which takes into account both model fit and complexity. We determined, for each participant, a ranking of all 20 models based on the BIC. The three rightmost columns of Table 1 show for each model: the number of participants for which the model was preferred over other models, the average BIC ranking 
Table 1 Model Comparison

\begin{tabular}{|c|c|c|c|c|c|c|c|}
\hline Model & $\mathrm{a}$ & $\mathrm{B}$ & $\mathrm{v}$ & Ter & n BIC Best & av. BIC rank & av. model weight \\
\hline 1 (Winner) & $\mathrm{p}$ & $\mathrm{p}, \mathrm{r}$ & $\mathrm{p}, \mathrm{s}$ & - & 26 & 2.16 & 0.62 \\
\hline 2 & $\mathrm{p}$ & $\mathrm{p}$ & $\mathrm{p}, \mathrm{r}, \mathrm{s}$ & $\mathrm{p}, \mathrm{r}$ & 3 & 6.47 & 0.06 \\
\hline 3 & $\mathrm{p}$ & $\mathrm{p}$ & $\mathrm{p}, \mathrm{r}, \mathrm{s}$ & - & 0 & 10.49 & 0 \\
\hline 4 & $\mathrm{p}$ & $\mathrm{p}$ & $\mathrm{p}, \mathrm{s}$ & - & 2 & 5.95 & 0.05 \\
\hline 5 & $\mathrm{p}$ & $\mathrm{p}, \mathrm{r}$ & $\mathrm{p}, \mathrm{r}, \mathrm{s}$ & $\mathrm{p}, \mathrm{r}$ & 0 & 17.09 & 0 \\
\hline 6 & $\mathrm{p}$ & $\mathrm{p}, \mathrm{r}$ & $\mathrm{p}, \mathrm{r}, \mathrm{s}$ & - & 0 & 16.44 & 0 \\
\hline 7 & $\mathrm{p}$ & $\mathrm{p}, \mathrm{r}$ & $\mathrm{p}, \mathrm{r}, \mathrm{s}$ & - & 0 & 14 & 0 \\
\hline 8 & $\mathrm{p}$ & $\mathrm{p}, \mathrm{r}$ & $\mathrm{p}, \mathrm{s}$ & - & 0 & 8.6 & 0 \\
\hline 9 & $\mathrm{p}$ & $\mathrm{p}, \mathrm{r}$ & $\mathrm{p}, \mathrm{s}: \mathrm{sym}$ & - & 3 & 8.74 & 0.04 \\
\hline 10 & $\mathrm{p}$ & $\mathrm{p}, \mathrm{r}$ & $\mathrm{s}$ & - & 3 & 9.51 & 0.07 \\
\hline 11 & $\mathrm{p}, \mathrm{r}$ & $\mathrm{p}$ & $\mathrm{p}$ & - & 1 & 7.73 & 0.01 \\
\hline 12 & $\mathrm{p}, \mathrm{r}$ & $\mathrm{p}$ & $\mathrm{p}, \mathrm{r}, \mathrm{s}$ & $\mathrm{p}, \mathrm{r}$ & 0 & 14.93 & 0 \\
\hline 13 & $\mathrm{p}, \mathrm{r}$ & $\mathrm{p}$ & $\mathrm{p}, \mathrm{r}, \mathrm{s}$ & - & 0 & 11.74 & 0.01 \\
\hline 14 & $\mathrm{p}, \mathrm{r}$ & $\mathrm{p}$ & $\mathrm{p}, \mathrm{s}$ & - & 0 & 7.92 & 0 \\
\hline 15 & $\mathrm{p}, \mathrm{r}$ & $\mathrm{p}, \mathrm{r}$ & $\mathrm{p}, \mathrm{r}, \mathrm{s}$ & $\mathrm{p}, \mathrm{r}$ & 0 & 18.63 & 0 \\
\hline 16 & $\mathrm{p}, \mathrm{r}$ & $\mathrm{p}, \mathrm{r}$ & $\mathrm{p}, \mathrm{s}$ & - & 0 & 10.34 & 0 \\
\hline 17 & $\mathrm{p}, \mathrm{r}$ & $\mathrm{p}, \mathrm{r}$ & $\mathrm{p}, \mathrm{s}$ & - & 0 & 10.67 & 0.01 \\
\hline 18 & $\mathrm{p}, \mathrm{r}$ & $\mathrm{p}, \mathrm{r}$ & $\mathrm{p}, \mathrm{s}$ & - & 0 & 10.31 & 0 \\
\hline 19 & $\mathrm{p}, \mathrm{r}$ & $\mathrm{p}, \mathrm{r}$ & $\mathrm{p}, \mathrm{s}: \mathrm{sym}$ & - & 5 & 8.09 & 0.1 \\
\hline 20 & $\mathrm{p}, \mathrm{r}$ & $\mathrm{p}, \mathrm{r}$ & $\mathrm{s}$ & - & 0 & 10.16 & 0.01 \\
\hline
\end{tabular}

Note. Comparison of models with different parameter constellations. Each column shows the effects of the different models allowed on parameters $\mathrm{a}=$ boundary separation, $\mathrm{B}=$ response Bias, $\mathrm{v}=$ drift rate, and Ter $=$ non-decision time. Effects: p: perceptual-preferential, r: risk level, s: stimulus type, s:sym: symmetric effect of stimuli. Rightmost columns show the number of participants for which the model was preferred over other models, the average BIC ranking across participants, and the average model weight over participants

across participants, and the average Bayesian model weight over participants. These model weights can be interpreted as posterior probabilities for all models (given equal prior probabilities, Raftery, 1999).

The model that performed best in describing the behavior in all conditions is model 1 (Winner model) of Table 1 . This model outperformed the other models that we fitted for 26 out of 43 participants and ended within the first three ranked models for 35 out of 43 participants. For the eight participants for which the winner model had a lower rank, the best fitting models were model 4 (2 pp), model 9 ( $3 \mathrm{pp}$ ), model $10(1 \mathrm{pp})$ and model $20(2 \mathrm{pp})$. These results show that the consistency of the model comparison across participants is rather strong. Therefore, we discuss the effects of the relevant factors on the diffusion model parameters as captured by the Winner model.

\section{Model fit}

Figure 4 shows the predictions of the Winner model plotted against the observed data. The upper panels show the fit of the RT quantiles $(.1, .3, .5, .7$, and .9) for majority responses (correct/EV-maximizing). The grey areas represent the observed RT quantiles (medians over participants), where the width of the area represents +/- one standard error. In the background, the white area and black lines represent the median of observed and predicted minority responses (errors/EV-minimizing). Similarly, in the lower panels, the grey areas represent the median response proportions +/one standard error. The white lines show the median model predictions, with error bars representing +/- one standard error. Overall, we observe a rather good fit of both the choice proportions and the 5 RT quantiles. However, the .9 quantile of RT is rather strongly overestimated by the model for the $50 \%$ stimuli, in particular in the perceptual task. We inspected the fit of more flexible models and found similar misfits. A very plausible explanation for this misfit is the fact that in the perceptual task, the $50 \%$ stimuli contain no information about the correct answer, because there is no correct answer. This yields guessing choice accuracy, which is captured by the diffusion model by a zero drift rate. A zero drift rate results in very slow predicted RTs. However, participants might feel the urgency to respond and might therefore decide to guess when they realize that there is no evidence in the stimulus. This leads to responses that are faster than predicted. Currently, several models are developed that include an urgency mechanism to account for this phenomenon (Cisek, Puskas \& El-Murr, 2009; Gluth et al., 

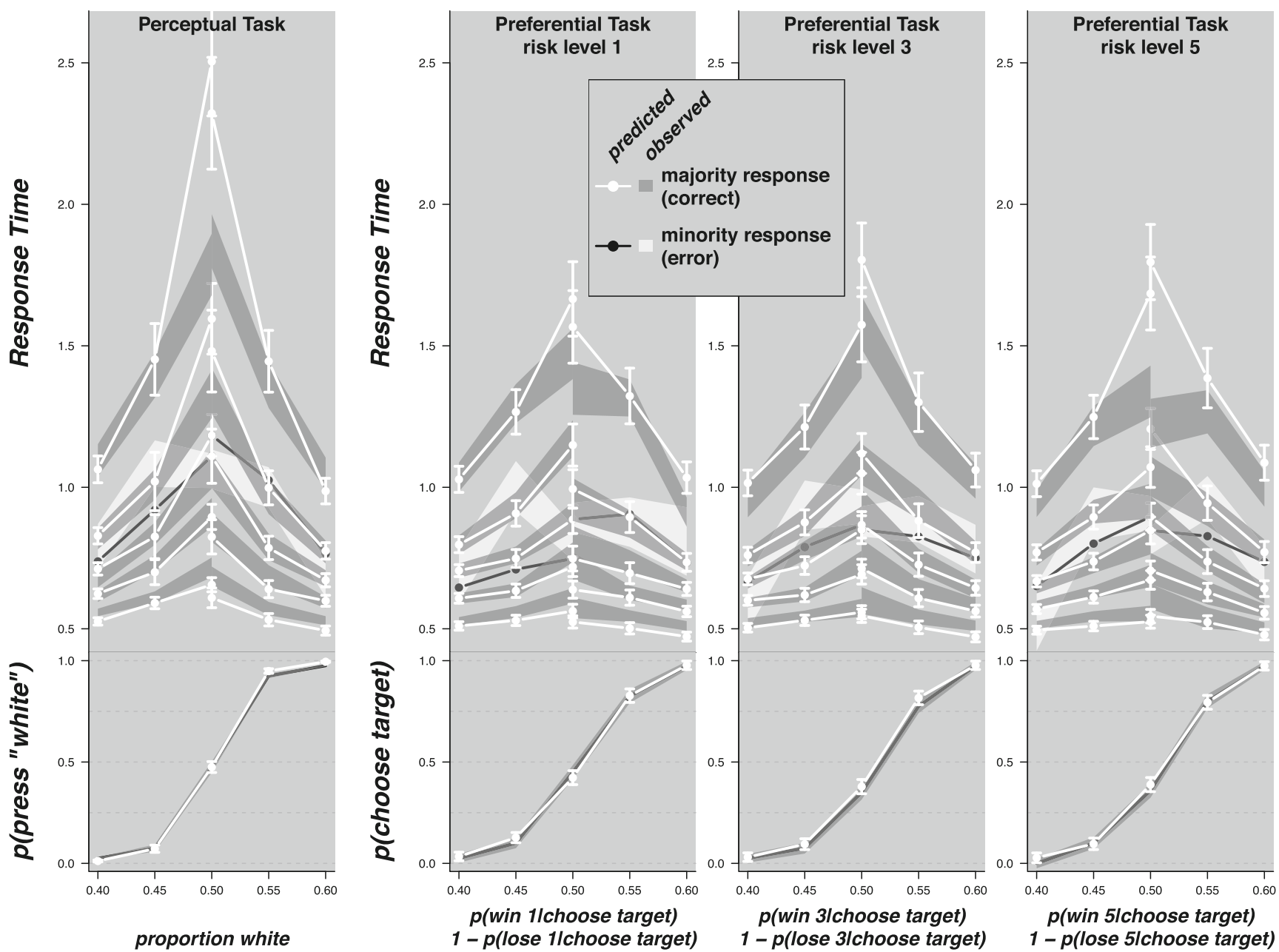

Fig. 4 Observed and predicted .1, .3, .5, .7, .9 RT quantiles and choice proportions. Majority (correct, EV-optimizing) responses: The white lines show model predictions with error bars containing \pm one $\mathrm{SE}$, grey areas show observed data \pm one SE. Minority (error, EVminimizing) responses: The black line shows model predictions with error bars containing \pm one SE, white areas show observed data \pm one SE. Note that for these minority responses, only the .5 quantile is shown. Because for 50/50 stimuli there is no majority response, both responses are displayed
2012). The application of such models is beyond the scope of this paper.

\section{Diffusion model inference: perceptual versus preferential choices}

In the Winner model, the difference between perceptual and preferential decision making was accounted for by leaving drift rate, boundary separation and response bias free to vary between the two conditions. The leftmost panels of Fig. 5 show that the boundary separation parameter was for most participants estimated to be lower in the preferential than the perceptual condition. This effect on boundary separation shows that most participants responded more cautiously making perceptual than making preferential decisions. Note, however, that the distribution of this effect, as plotted in the lower left panel, has a peak close to zero, but a large tail to the left. This suggests that most participants made perceptual decisions slightly more cautiously, whereas a minority showed more dramatic effects.

The middle two panels of Fig. 5 show that participants had on average no response bias in the perceptual task. However, in the preferential version of the task, participants had on average a slight a priori preference for the presented, ambiguous gamble. This shift in bias could be interpreted as an indication of an a priori ambiguity-loving attitude. An alternative explanation, however, is offered in the discussion section.

Finally, the average drift rate appeared lower in the preferential than in the perceptual task, most strongly expressed when the evidence in favor of the ambiguous gamble was at its strongest, i.e., at the 60/40 stimulus. This effect 
suggests that participants processed the stimulus information in a more deterministic way when forming a perceptual judgement than when making a preferential judgement.

This combination of parameter effects accounts for the differences on choice proportion and RT between the perceptual task and the preferential task. Note that, although participants accumulated evidence at a higher rate in the perceptual task (as expressed in the drift rate), participants did not respond quicker in this task. The fact that participants respond slower in the perceptual version of the task is entirely due to an increased boundary separation, that is, they responded more cautiously.

\section{Diffusion model inference: effects of risk level}

The Winner model further shows an effect of the different risk levels on behavior: increasing the risk level shifts participants' response bias. The middle panels of Fig. 5 show that, relative to the equal risk condition, the average response bias shifts toward the 50/50-reference gamble when risk level is increased. Note however that the distributional plots in the lower panel of Fig. 5 shows a sizable spread for both effects (Risk 1 vs. Risk 3, and Risk 3 vs. Risk 5) that entails zero. These observations suggest that most but not all participants responded in a way consistent with risk aversion rather than risk seeking in this task.

\section{Diffusion model inference: effects of the proportion of white and black marbles}

The Winner model captures the effect of different proportions of white and black marbles by the drift rate. The upper right panel of Fig. 5 shows an asymmetric effect of the proportion of white and black marbles. Indeed, the model allowing for such an asymmetric effect outperformed models that restricted the stimulus effect to be symmetric. This result shows that stimulus information disadvantageous for the target gamble on average leads to a stronger drift rate than stimulus information favoring the target gamble. This effect implies that participants value negative information about a gamble more heavily than positive information.

So, the difference between perceptual and preferential choice was captured by the boundary separation parameter of the diffusion model, suggesting that participants responded more cautiously in the perceptual task. In addition, in the preferential task, negative information about a gamble led to stronger drift rates than positive information, suggesting that participants value negative information
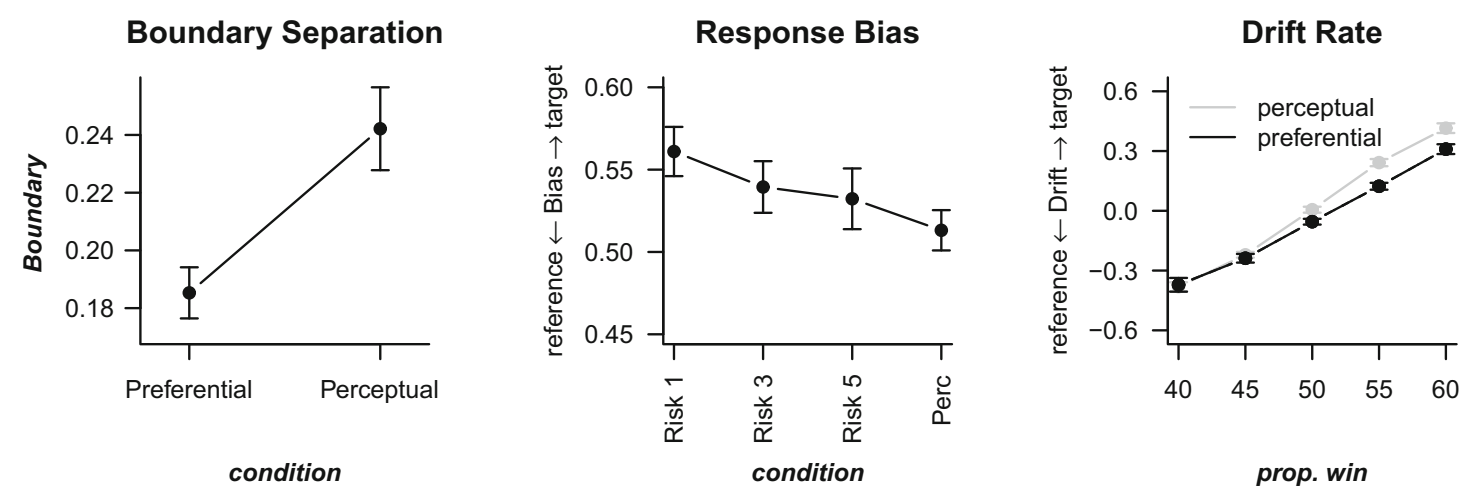
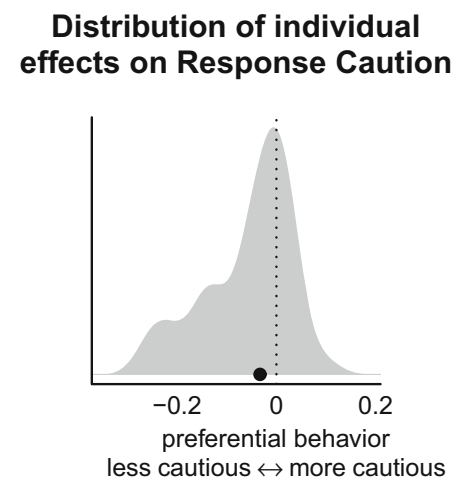
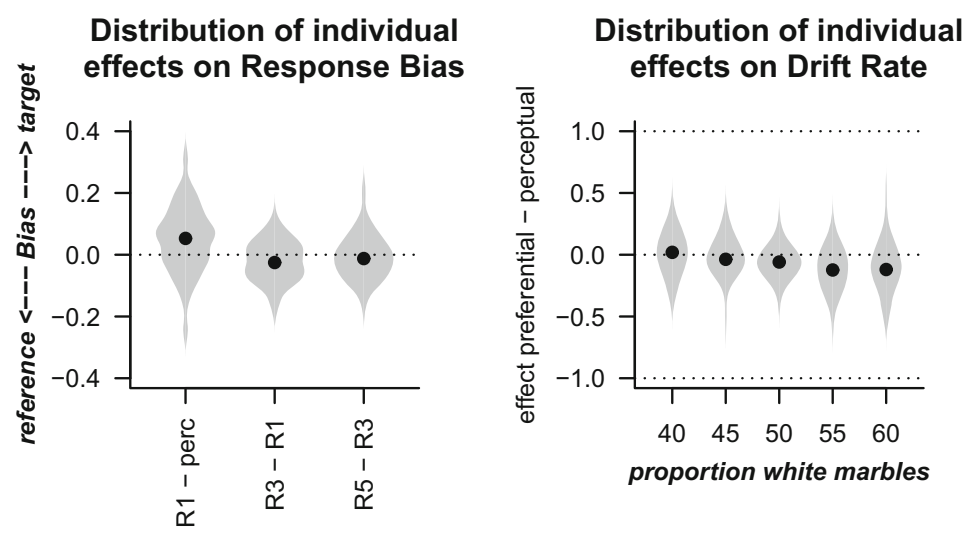

Fig. 5 Diffusion model parameters based on the Winner model. (Upper panel) show over-participants means of parameter estimates. (Lower panels) show the over-participants distribution of the effect for different contrasts. Solid dots represent the median effects 
more strongly than positive information. Further, increasing the risk level of the target gamble made participants shift their response bias toward the reference gamble, indicating risk aversion.

\section{Discussion}

In this paper, we described perceptual and preferential decisions with the same sequential sampling model. By doing so we could identify the crucial differences between the two types of decision making. First, we discussed the similarities and differences between perceptual and preferential decisions on theoretical grounds. Second, we developed a new choice paradigm which uses the same stimulus material for perceptual and preferential decisions. The experiment using this marbles task showed that in the perceptual task, people process information more deterministically and behaved more cautiously than in the preferential task. Finally, we found that participants' risk aversion was reflected in a shift of response bias.

\section{Our findings in theoretical perspective}

First, our results show that, although the exact same stimuli were used in the two versions of the task, slower evidence accumulation took place in the preferential as compared to the perceptual task. This finding might reflect the fact that in the perceptual task, where the outcomes are deterministic, the relative choice preference resulting from samples from the stimulus is stronger than in the preferential case, where outcomes are variable in nature. In particular, we found that positive information in the target gamble (more than $50 \%$ white) led to lower rates of information accumulation than negative information. This effect seems to be in line with the way in which loss aversion is built into the leaky competing accumulator model by Usher \& McClelland (2004). This model describes loss aversion as inherent in the valuation process, as opposed to a prior bias against options with potential losses.

Second, our results show that participants responded with more caution in the perceptual than in the risky preferential task. At first sight, this seems an unexpected finding, since the intuition is that a decision maker needs time to elaborate about the potential risks and benefits of preferential choice options over and above the time needed for perceptual processing. However, there is a clear explanation for this finding. In the perceptual version of our task, a correct answer always yields a gain of one unit of money; an error yields a loss of one unit. So, the difference in expected payoff for choosing one or the other option is two units of money. In the preferential version of the task, the biggest difference in expected payoff happens in the case of a $60 / 40$ target gamble. The average payoff when choosing this target gamble is $.6 \times 1+.4 \times-1$ is .2 units of money (minus . 2 for a $40 / 60$ gamble). The other option, the reference gamble, has an average payoff of zero. Thus, the difference in expected payoff for choosing the target or reference gamble is only .2 units here, that is 10 times as little as in the perceptual case. Given this difference in incentive, it is plausible that participants respond more carefully in the perceptual version of the task. In other words, when people are forced to choose between decision options that contain some inevitable risk they make their decisions less carefully. An interesting question for future research is whether participants would also respond less carefully in a perceptual task when the payoff is defined probabilistically. In sum, these results seem consistent with past research showing that increased monetary incentives motivate people to make decision more carefully in the preferential choice situations (Smith \& Walker, 1993) as well as in cases where an objective criterion for choice accuracy is present (Touron, Swaim \& Hertzog, 2007).

There is, however, an alternative explanation for lower response caution in the preferential task. When participants aim at responding at a similar pace during both tasks and their rate of information processing is lower in the preferential task (as we find), similar pace can be accomplished by setting less conservative response boundaries (Bogacz, Brown, Moehlis, Holmes \& Cohen, 2006).

Third, participants behaved on average risk-averse: When the risk of the target gamble was increased relative to the reference gamble, participants shifted their a priori bias in the direction of the reference gamble.

Fourth, participants were on average ambiguity-seeking; When deciding between two gambles with equal outcomes (risk level 1) participants had on average a bias towards the unknown reference gamble. Possibly, the low amounts one could win or lose in this task made participants particularly willing to go for the unknown. Note that another interpretation of this effect is that participants prefer the target gamble because they see it on each trial and do not need to be remember it like they have to remember the 50/50 reference gamble. Interestingly, the effect was not detected in the analyses of choice proportions. This illustrates the ability of the diffusion model to detect effects that are not manifest in the raw data (White, Ratcliff, Vasey \& McKoon, 2010). It should be noted here that we did not counterbalance the button mapping, so that this effect could also result from an a priori tendency to press right over left.

The model that performed best did not include an effect on the non-decision time parameter. We inspected the parameter estimates of the full model (model 16) and found no consistent difference in non-decision time between the perceptual and preferential task. This is interesting, since non-decision time is assumed to capture the encoding of information. In the preferential task, participants need to 
encode additional information, i.e., the payoffs. The fact that we do not find an effect on non-decision time suggests that participants encoded this payoff information when it was presented at the start of the trial blocks and not during each individual trial.

\section{Limitations of this study}

We find that people respond slightly quicker in the preferential as compared to the perceptual task. This finding might appear counter-intuitive: in real life, people presumably spend more time on making preferential decisions (e.g., choosing a vacation destination) than on perceptual decisions (e.g., judging the color of a traffic light). However, many less important preferential decisions such as choosing an ice cream do not require much time either. Thus, the time spent on deliberation depends on the type of preferential decision. When the goals are complex (e.g., when choosing between vacation destinations) people spend more time than when the goals are clear (e.g., when choosing between gambles). The preferential version of our marbles task is situated at the latter extreme. In this task, we made the preferential decision situation so simple, that we minimized the difference between the two types of decision making. The question remains how well our results generalize towards more complex preferential decision making situations.

Future work should build upon the current findings to study preferential decisions that go beyond the simple risky gamble choices that we studied here. One such attempt is made in the work by Polanía, Krajbich, Grueschow \& Ruff (2014). In their study, participants were presented with two pictures of food items. In the preferential choice condition, participants choose which item they preferred. In the perceptual choice condition, participants were to judge which of two items covered more of the background of the display. This procedure offers a preferential choice situation that goes beyond a simple risky choice situation requiring participants to trade-off the different attributes of the food items (i.e., tastiness vs. healthiness). It lacks however the quality of the marbles task that the exact same stimulus property (the relative proportion of black and white marbles) is used to make either a preferential or a perceptual decision.

\section{Current perspectives on perceptual and preferential decision making}

We are certainly not the first to shed light on the commonalities of perceptual and preferential decision making. The study we report here should be seen in perspective of rather diverse endeavors that have recently been made on this topic. For example, the existence of shared underlying physiological principles for both types of decisions has been studied theoretically and empirically by Summerfield \& Tsetsos (2012) and Polanía et al. (2014). More directly, our results relate to studies by Busemeyer (1985), Tsetsos, Chater \& Usher (2012) and Zeigenfuse et al. (2014). In contrast to our method, in which participants have to infer from the stimuli the probability of each of two possible outcomes, these studies presented participants with samples of a continuous distribution. ${ }^{2}$ In particular, Zeigenfuse et al. (2014) compared perceptual and preferential choices in a similar way as we do here. However, they presented an outcome distribution as a flashing aperture in which the number of dots in each flash represented a sample from that distribution. Participants had to choose between a draw from this distribution or a sure number. Choice behavior on this task was compared to behavior of participants when they were asked to decide whether the average of the flashing distribution was higher or lower than a presented number. In line with our results, Zeigenfuse et al. (2014) found that behavior is more directly determined by the stimulus in the perceptual version of the task. However, in their diffusion model analyses, this was accounted for by just a higher rate of information accumulation for perceptual choices. In contrast, we find not only an increased rate of information accumulation but also an increased boundary separation. More crucially, Zeigenfuse et al. (2014, page 185) conclude that participants "appear to weigh experienced events in an optimistic manner", whereas in our study, it seems that participants value negative information more strongly than positive information. Future research should address the question as to what factors yield pessimistic or optimistic information integration.

\section{Towards an integrated account of perceptual and preferential choice}

Sequential sampling models were originally introduced to describe the processes underlying perceptual decision making (Link and Heath, 1975; Ratcliff, 1978). More recently, sequential sampling models have received increasing attention in the preferential decision making literature (Busemeyer \& Townsend, 1993; Roe, Busemeyer \& Townsend, 2001; Gluth et al., 2012; Usher \& McClelland, 2001). These models for perceptual and preferential decision making share the assumption of sequential sampling. In both the perceptual and preferential domain, this simple principle accounts for important empirical phenomena described in the literature. The question rises to what degree the two types of decision making actually rely on the same processes. As Gold \& Shadlen (2007) argue, the noise of

\footnotetext{
${ }^{2}$ In fact, the distributions of outcomes in these studies were approximately continuous.
} 
information accumulation that is central to perceptual decision making might very well yield the probabilistic nature of preferential choice. At the same time they argue that the judgment of value that is central to preferential decision making plays a role as well in perceptual choice, for example when a decision maker values speed vs. accuracy incentives.

We view the study that we present here as a step up to further integrate our knowledge about perceptual and preferential choice. The fact that both domains of decision making have been modeled with very similar models, suggests the possibility to describe both types of behavior within one model. Our conclusions set out criteria that an integrated model should comply with. Current work focuses on explicitly implementing a valuation function at the input side of the diffusion process in order to explain perceptual and preferential choice within the same framework.

\section{References}

Bogacz, R., Brown, E., Moehlis, J., \& Holmes, P. (2006). The physics of optimal decision making: A formal analysis of models of performance in two-alternative forced choice tasks. Psychol. Rev., 113, 700-765.

Britten, K.H., Shadlen, M.N., Newsome, W.T., \& Movshon, J. A. (1992). The analysis of visual motion: A comparison of neuronal and psychophysical performance. J. Neurosci., 12, 47454765.

Busemeyer, J.R. (1985). Decision making under uncertainty: A comparison of simple scalability, fixed-sample, and sequentialsampling models. Journal of Experimental Psychology: Memory, and Cognition, 11, 538-564.

Busemeyer, J. R., \& Townsend, J. T. (1993). Decision field theory: A dynamic-cognitive approach to decision making in an uncertain environment. Psychol. Rev., 100, 432-459.

Cisek, P., Puskas, G. A., \& El-Murr, S. (2009). Decisions in changing conditions: The urgency-gating model. The Journal of Neuroscience, 29, 11560-11571.

Diederich, A., \& Busemeyer, J. R. (2003). Simple matrix methods for analyzing diffusion models of choice probability choice response time, and simple response time. J. Math. Psychol., 47, 304322.

Dutilh, G., Krypotos, A.M., \& Wagenmakers, E. J. (2011). TaskRelated vs. Stimulus-Specific Practice: A Diffusion Model Account. Exp. Psychol., 58, 434-442.

Ellsberg, D. (1961). Risk, ambiguity, and the Savage axioms. Q. J. Econ., 643-669.

Gluth, S., Rieskamp, J., \& Büchel, C. (2012). Deciding when to decide: Time-variant sequential sampling models explain the emergence of value-based decisions in the human brain. The Journal of Neuroscience, 32, 10686-10698.

Gold, J. I., \& Shadlen, M. N. (2007). The neural basis of decision making. Annu. Rev. Neurosci., 30, 535-574.

Holt, C. A., \& Laury, S. K. (2002). Risk aversion and incentive effects. Am. Econ. Rev., 92, 1644-1655.

Kahneman, D., \& Tversky, A. (1979). Prospect theory: An analysis of decision under risk. Journal: Econometrica, 47, 263-291.

Keuleers, E., Diependaele, K., \& Brysbaert, M. (2010). Practice effects in large-scale visual word recognition studies: A lexical decision study on 14,000 Dutch mono-and disyllabic words and nonwords. Front. Psychol.

Link, S. W., \& Heath, R. A. (1975). A sequential theory of psychological discrimination. Psychometrika, 40, 77-105.

Milosavljevic, M., Malmaud, J., Huth, A., Koch, C., \& Rangel, A. (2010). The Drift Diffusion Model can account for the accuracy and reaction time of value-based choices under high and low time pressure. Judgment and Decision Making, 5, 437-449.

Morey, R. D., Rouder, J. N., \& Jamil, T. (2014). BayesFactor: Computation of Bayes factors for common designs. R package version 0.97. http://CRAN.R-project.org/package=BayesFactor

Neumann, J., \& von Morgenstern, O. (1945). Theory of games and economic behavior. Bull. Am. Math. Soc., 51, 498-504.

Pachella, R.G. (1974). The interpretation of reaction time in information-processing research. In B.H. Kantowitz (Ed.), Human information processing: Tutorials in performance and cognition (pp. 41-82). Lawrence Erlbaum Associates: Hillsdale (NJ).

Pettibone, J. C., \& Wedell, D. H. (2000). Examining models of nondominated decoy effects across judgment and choice. Organ. Behav. Hum. Decis. Process., 81, 300-328.

Phillips, L.D., \& Edwards, W. (1966). Conservatism in a simple probability inference task. J. Exp. Psychol., 72, 346-354.

Polanía, R., Krajbich, I., Grueschow, M., \& Ruff, C. C. (2014). Neural oscillations and synchronization differentially support evidence accumulation in perceptual and value-based decision making. Neuron, 82, 709-720.

Rae, B., Heathcote, A., Donkin, C., Averell, L., \& Brown, S. (2014). The hare and the tortoise: Emphasizing speed can change the evidence used to make decisions. J. Exp. Psychol. Learn. Mem. Cogn., 40, 1226-1243.

Raftery, A. E. (1999). Bayes factors and BIC Bayes factors and BIC. Sociol. Methods Res., 27, 411-417.

Ratcliff, R. (1978). A theory of memory retrieval. Psychol. Rev., 85, 59-108.

Ratcliff, R., \& Rouder, J. N. (1998). Modeling response times for twochoice decisions. Psychol. Sci., 9, 347-356.

Ratcliff, R., \& Rouder, J. N. (2000). A diffusion model account of masking in two-choice letter identification. J. Exp. Psychol. Hum. Percept. Perform., 26, 127-140.

Ratcliff, R., \& Tuerlinckx, F. (2002). Estimating parameters of the diffusion model: Approaches to dealing with contaminant reaction times and parameter variability. Psychon. Bull. Rev., 9, 438-481.

Rieskamp, J., Busemeyer, J. R., \& Mellers, B. A. (2006). Extending the bounds of rationality: Evidence and theories of preferential choice. J. Econ. Lit., 44, 631-661.

Roe, R. M., Busemeyer, J. R., \& Townsend, J. T. (2001). Multialternative decision field theory: A dynamic connectionist model of decision making. Psychol. Rev., 108, 370-392.

Savage, L. J. (1954). The foundation of statistics. Wiley.

Schouten, J. F., \& Bekker, J. A. M. (1967). Reaction time and accuracy. Acta Psychol., 27, 143-153.

Smith, V.L., \& Walker, J. M. (1993). Rewards, experience and decision costs in first price auctions. Econ. Inq., 31, 237-244.

Summerfield, C., Egner, T., Mangels, J., \& Hirsch, J. (2006). Mistaking a house for a face: Neural correlates of misperception in healthy humans. Cereb. Cortex, 16, 500-508.

Summerfield, C., \& Tsetsos, K. (2012). Building bridges between perceptual and economic decision-making: Neural and computational mechanisms. Front. Neuroendocrinol., 6.

Thapar, A., Ratcliff, R., \& McKoon, G. (2003). A diffusion model analysis of the effects of aging on letter discrimination. Psychol. Aging, 18, 415-429.

Touron, D. R., Swaim, E. T., \& Hertzog, C. (2007). Moderation of older adults' retrieval reluctance through task instructions and 
monetary incentives. The Journals of Gerontology Series By: Psychological Sciences and Social Sciences, 62, 149-155.

Tsetsos, K., Chater, N., \& Usher, M. (2012). Salience driven value integration explains decision biases and preference reversal. In Proceedings of the National Academy of Sciences (Vol. 109, pp. 9659-9664).

Tversky, A., \& Kahneman, D. (1992). Advances in prospect theory: Cyumulative representation of uncertaint. J. Risk Uncertain., 5, 297-323.

Usher, M., \& McClelland, J. L. (2001). The time course of perceptual choice: The leak, competing accumulator model. Pshychological Review, 108, 550-592.

Usher, M., \& McClelland, J. L. (2004). Loss aversion and inhibition in dynamical models of multialternative choice. Psychol. Rev., 111, 757-769.

van Ravenzwaaij, D., Dutilh, G., \& Wagenmakers, E. J. (2012). A diffusion model decomposition of the effects of alcohol on perceptual decision making. Psychopharmacology, 219, 1017-1025.

Van Ravenzwaaij, D., Van der Maas, H.L. J., \& Wagenmakers, E.J. (2011). Does the name-race implicit association test measure racial prejudice? Exp. Psychol., 58, 271-277.
Vandekerckhove, J., \& Tuerlinckx, F. (2007). Fitting the Ratcliff diffusion model to experimental data. Psychon. Bull. Rev., 14, 10111026.

von Neumann, J., \& Morgenstern, O. (1945). Theory of games and economic behavior. Bull. Am. Math. Soc., 51, 498-504.

Voss, A., Rothermund, K., \& Voss, J. (2004). Interpreting the parameters of the diffusion model: An empirical validation. Mem. Cogn., $32,1206-1220$.

Wagenmakers, E. J., Ratcliff, R., Gomez, P., \& McKoon, G. (2008). A diffusion model account of criterion shifts in the lexical decision task. J. Mem. Lang., 58, 140-159.

Weber, E. U., \& Johnson, E. J. (2009). Mindful judgment and decision making. Annu. Rev. Psychol., 60, 53-85.

White, C. N., Ratcliff, R., Vase, M. W., \& McKoon, G. (2010). Using diffusion models to understand clinical disorders. J. Math. Psychol., 39, 96-52.

Wickelgren, W. A. (1977). Speed-accuracy tradeoff and information processing dynamics. Acta Psychol., 41, 67-85.

Zeigenfuse, M.D., Pleskac, T. J., \& Liu, T. (2014). Rapid decisions from experience. Cognition, 131, 181-194. 